\title{
Effectiveness of a Wearable Fitness Tracker: Practice Implications in Allied Health - a Single Case Study
}

Thomas William Miller

University of Connecticut - Storrs, tom.miller@uconn.edu

Follow this and additional works at: https://nsuworks.nova.edu/ijahsp

Part of the Medicine and Health Sciences Commons

\section{Recommended Citation}

Miller TW. Effectiveness of a Wearable Fitness Tracker: Practice Implications in Allied Health -- a Single Case Study. The Internet Journal of Allied Health Sciences and Practice. 2017 Jan 01;15(1), Article 3.

This Case Study is brought to you for free and open access by the College of Health Care Sciences at NSUWorks. It has been accepted for inclusion in Internet Journal of Allied Health Sciences and Practice by an authorized editor of NSUWorks. For more information, please contact nsuworks@nova.edu. 


\title{
Effectiveness of a Wearable Fitness Tracker: Practice Implications in Allied Health - a Single Case Study
}

\begin{abstract}
ABSTRACT

Purpose: The purpose of this single case study was to examine the effectiveness of utilizing a wearable fitness tracker device in self-monitoring behavior change in complying with prescribed treatment. It was hypothesized that using a wearable self-monitoring device while involved in the treatment of multiple medical conditions will be beneficial to behavioral compliance and behavioral change in an overweight type 2 diabetic, geriatric subject being treated by a multidisciplinary health team utilizing an integrated treatment model.
\end{abstract}

Methods: An exploratory single case study research design is employed to explore those situations in which the intervention, a wearable monitoring device, is employed. This observational case study model was applied to the utility of self-monitoring wearable smart technology marketed as FitBit for selfmonitoring activity and exercise over a 36-week period.

Results: After the 36-week intervention program, results revealed qualitative improvements in the (1) active minutes, (2) steps taken and (3) miles walked by the subject. In addition, the technology reported on calories burned, sleep hours and minutes logged and liquid consumed during each 24-hour period. The integrated allied health team was able to monitor changes made over time and noted improved time dedicated to exercise, walking and total miles walked. On miles walked per day, the results show that the subject increased miles walked from less than one mile per day to more than 4.6 miles per day which approached the recommended 5 miles per day goal. The calorie monitor aided the subject in changing calorie intake from that which exceeded 3500 per day to the recommended 2500 calories per day. Furthermore, the hours slept per night changed from less than 6.8 per night to 8.1 per day.

Conclusions: It was concluded that a 36-week intervention program can be an effective intervention methodology for improved health and well-being as measured by standard healthcare and wellness markers utilizing a wearable fitness tracker device like the Fitbit. While further research is needed with a larger sample, it is therefore recommended that allied health professionals consider the utilization of wearable smart technology as an adjunct to self-monitoring and compliance with treatment planning and follow-up care with allied health care providers.

\section{Author Bio(s)}

Thomas W. Miller PhD, ABPP is Professor Emeritus and Senior Research Scientist at the Institute for Health, Intervention, and Policy, University of Connecticut, and also Professor, Department of Gerontology, College of Public Health and Department of Psychiatry, College of Medicine, University of Kentucky.

Robert F. Kraus MD is Professor and former chair for the Department of Psychiatry, College of Medicine, and Professor, Department of Anthropology, College of Arts and Sciences, University of Kentucky.

Deborah Burton MA, $\mathrm{PhD}(\mathrm{c})$ is the manager for Telehealth, Strategy, and Business Development at Catholic Health Network, Kentucky One Health, Lexington, Kentucky.

Christina Busse, MS, RD, LD is a Registered Dietitian for Community Outreach and Transitions of Care, at Catholic Health Network, Kentucky One Health, Lexington, Kentucky. 


\section{Acknowledgements}

The authors wish to acknowledge the assistance from Jill Livingston, Research Librarian, University of Connecticut.ge for her assistance with this study. 


\title{
TIAHSP \\ The Internet Joưnal of Allied Health Sciences and Practice
}

Dedicated to allied health professional practice and education

Vol. 15 No. 1 ISSN 1540-580X

\section{Effectiveness of a Wearable Fitness Tracker: Practice Implications in Allied Health - a Single Case Study}

\author{
Thomas William Miller, $\mathrm{PhD}^{1}$ \\ Robert F. Kraus, MD 1 \\ Deborah Burton, MA, PhDc 2,3 \\ Christina Busse, MS, RD, LD ${ }^{3}$ \\ 1. University of Connecticut \\ 2. University of Kentucky \\ 3. Kentucky One Health \\ United States
}

\begin{abstract}
Purpose: The purpose of this single case study was to examine the effectiveness of utilizing a wearable fitness tracker device in self-monitoring behavior change in complying with prescribed treatment. It was hypothesized that using a wearable self-monitoring device while involved in the treatment of multiple medical conditions will be beneficial to behavioral compliance and behavioral change in an overweight type 2 diabetic, geriatric subject being treated by a multidisciplinary health team utilizing an integrated treatment model.

Methods: An exploratory single case study research design is employed to explore those situations in which the intervention, a wearable monitoring device, is employed. This observational case study model was applied to the utility of self-monitoring wearable smart technology marketed as FitBit for self-monitoring activity and exercise over a 36-week period.

Results: After the 36-week intervention program, results revealed qualitative improvements in the (1) active minutes, (2) steps taken and (3) miles walked by the subject. In addition, the technology reported on calories burned, sleep hours and minutes logged and liquid consumed during each 24 -hour period. The integrated allied health team was able to monitor changes made over time and noted improved time dedicated to exercise, walking and total miles walked. On miles walked per day, the results show that the subject increased miles walked from less than one mile per day to more than 4.6 miles per day which approached the recommended 5 miles per day goal. The calorie monitor aided the subject in changing calorie intake from that which exceeded 3500 per day to the recommended 2500 calories per day. Furthermore, the hours slept per night changed from less than 6.8 per night to 8.1 per day.

Conclusions: It was concluded that a 36-week intervention program can be an effective intervention methodology for improved health and well-being as measured by standard healthcare and wellness markers utilizing a wearable fitness tracker device like the Fitbit. While further research is needed with a larger sample, it is therefore recommended that allied health professionals consider the utilization of wearable smart technology as an adjunct to self-monitoring and compliance with treatment planning and follow-up care with allied health care providers.
\end{abstract}

\section{Introduction}

The allied health professions have well recognized that patient self-management and motivation are critical ingredients in all clinical healthcare. Support of patient self-management skill is viewed as a critical component in the effective management of chronic 
illness care and improved patient outcomes. Self-management may require more than traditional knowledge-based patient education to include processes that develop patient problem-solving skills and improve self-efficacy.

Emerging technologies are offering allied health professionals assistance in monitoring patient goal-setting, rewards and motivating patients to compete with their own bio-medical goals. Motivation is usually defined as the process that initiates, guides, and maintains goal-oriented behaviors. ${ }^{1}$ Motivation is often viewed as what causes a person to act on a goal in life. Motivation involves the biological, emotional, social, and cognitive forces that activate behavior. In everyday usage, the term motivation is frequently used to describe why a person does something.

Evidence-based research has demonstrated the utility of activity monitoring in ostensibly healthy adult and aging populations. ${ }^{1-5}$ The use of wearable devices has the potential to lead to positive health outcomes for patients with chronic disease. ${ }^{6}$ As populations age, there is increasing emphasis on reducing hospital stays, decreasing readmission rates, and helping patients self-manage their medical conditions in their own home environments. Perhaps activity monitoring using wearable smart technology can be an effective tool in such disease self-management programs.

Guidelines have been generated nationally to aid patients in setting goals for physical activity, caloric intake, good nutrition, and adequate sleep patterns. The U.S. Surgeon General's recommendation encourages 30-minutes of physical activity most days of the week and to achieve 10,000 steps per day is a recognized goal. ${ }^{7}$ Not everyone should start right out trying to achieve 10,000 steps per day. A meta-analysis of activity monitor-based intervention with diabetic patients concluded that activity monitoring had a significant and beneficial effect on physical activity, blood glucose, systolic blood pressure, body mass index, and improved biomarkers in disease management. ${ }^{6}$ Activity-monitoring devices that record walking behavior may be helpful in quantifying levels of ambulation and provide insights into important symptoms such as fatigue in multiple sclerosis patients. ${ }^{8}$ This research study found a significant relationship between the early recovery step count, hospital length of stay, and discharge disposition in a group of elderly cardiac surgery patients. The use of accelerometers and pedometers to measure physical activity has also enhanced treatments for individuals participating in pulmonary rehabilitation. 9-10

\section{METHODS}

An exploratory single case study research design was employed to assess the benefits of a wearable Smart technology and its use by allied health practitioners. This case study model is applied to self-monitoring associated with the use of a wearable smart technology device marketed as Fitbit for measuring physical activity, sleep patterns, and calorie intake and expenditure in an overweight, type 2 diabetic, geriatric subject.

\section{Study Design}

This is a single subject exploratory case study research design employed to measure specific variables identified by the treatment team. The use of a wearable Smart technology device marketed as FitBit is utilized to measure several variables related to health and wellness. This research study was approved by the Institutional Review Board (IRB) for the ethical treatment of human subjects at University of Connecticut - Storrs.

\section{Subject}

The subject, WT, is a 68-year-old male and retired teacher who presents with elevated lipids, high blood pressure, and a sedentary life style. He is a type 2 diabetic diagnosed for 22 years with complications from obesity, hypertension, and cardiovascular disease. He is 5'7" and weighs $268 \mathrm{lbs}$. with a body mass index of 35. The subject's blood pressure was $140 / 76$ and a pulse rate of 80 and regular. WT would benefit from increased physical activity, careful monitoring of calorie intake for his diabetes, coronary disease sleep monitoring, and other medical problems. At intake, WT indicates a desire to participate in a prescribed program of outpatient care working closely with the diabetes educator, registered dietician, health psychologist, and his physician to change his habits and improve his health and wellness profile.

After some discussion, WT showed an understanding of the benefits of using a self-monitoring device to gauge changes over time that addressed his weight, physical activity, caloric intake, sleep patterns, and water consumption. He agreed to increasing exercise on a graduated basis and agreed to work with a registered dietician to set goals that would achieve a nutritionally healthy meal plan.

The subject was issued a Fitbit One which is a wireless activity tracker that syncs using Bluetooth SMART technology on WT's personal smartphone. This technology permits the subject to record several daily activities, including but not limited to number of steps taken, distance travelled on foot, calories burned, vigorously active minutes, sleep efficiency, delicate movements during sleep, number of wake-ups during sleep, and other personal biomarkers.

(c) The Internet Journal of Allied Health Sciences and Practice, 2016 


\begin{abstract}
Measurements
Measurements of activities identified for the treatment plan were retrieved from a Fitbit which is an American-made product developed to measure biomarkers that include several personal metrics that include steps walked, activity time periods and quality of sleep. The Fitbit technology is worn on the non-dominant wrist and includes activity trackers. This type of wireless enabled wearable technology device measures and stores data that can be transmitted to allied health professionals. The Fitbit is a wireless activity tracker designed to sync using Bluetooth 4.0 or Bluetooth SMART technology. The wireless syncing is available on iOS and android devices. Fitbit records several daily activities, including but not limited to distance travelled on foot, number of floors climbed, calories burned, vigorously active minutes, sleep efficiency, delicate movements during sleep, number of wake-ups during sleep, and other personal data.
\end{abstract}

\title{
Procedures
}

In this single subject designed case study, the subject was trained to use, store, tabulate, self-enter certain data, and recharge the Fitbit. Specificity, self-entered data included how to enter the logging of food consumption, ADL activities, water intake, and weight monitoring, as well as tracking fitness goals throughout the day even while offline. Measurements are systematically synchronized to a Smartphone and Tablet with the appropriate app for storage, retrieval, and summary of data for the study.

\section{RESULTS}

Measurable results revealed that WT began to realize short term goals early on which served as a motivator for continued use. The initial benefit was a five-pound weight loss in the first two-week period. He continued to lose weight but at a slower rate during the first 12-week reporting period. At 36-weeks, he had lost a total of nearly 22 pounds. Much of this is attributed to his increased physical activity and caloric monitoring as evidenced by the results reported on the Smart technology.

Clinical staff including the physician, clinical registered dietician, diabetic educator, and health psychologist noted that in addition to the obvious benefits of enhanced self-esteem and healthier eating habits, this subject showed signs of enjoying some very important, perhaps life-saving, health benefits. His cholesterol lab tests were also reduced. After the 36-week intervention program, results revealed significant qualitative improvements in the active minutes, steps taken, and miles walked by the subject. In addition, the technology reported on calories burned, hours slept, and water consumed during each 24-hour period had achieved normal levels. The integrated allied health team was able to monitor changes made over time and noted improved time dedicated to exercise, walking and total miles walked.

On miles walked per day, the results show that the subject increased miles walked from less than one mile per day to more than 4.6 miles per day which approached the recommended 5 miles per day or 10,000 steps recommended by the Surgeon General. The calorie monitor aided the subject in changing calorie intake from that which exceeded 3500 calories per day to the recommended 2500 calories per day. Furthermore, the sleep total hours slept per night changed from less than 6.8 per night to 8.1 per day, which was recommended by his physician. The key markers for the study are summarized in Table 1.

Table 1: Measured Variables over 36-week study for Type 2 Diabetic Subject using Wearable Device

\begin{tabular}{|l|l|l|l|l|}
\hline \multicolumn{1}{|c|}{ Baseline } & \multicolumn{1}{c|}{ Measurements } & \multicolumn{1}{c|}{ 12 weeks } & \multicolumn{1}{c|}{ weeks } & \multicolumn{1}{c|}{ weeks } \\
\hline 8.1 & HbA1c & 7.8 & 7.3 & 6.3 \\
\hline 14 & Average Active Minutes/day & 23 & 48 & 64 \\
\hline$<1000$ & Average Steps Taken/day & 2991 & 6510 & 8931 \\
\hline$<1.00$ & Average Miles Walked/day & 1.39 & 3.03 & 4.15 \\
\hline NA & Calories Burned/day & 1.571 & 2,581 & 2,758 \\
\hline NA & Sleep hours \& minutes/night & $6 \mathrm{hr} 5 \mathrm{~min}$ & $6 \mathrm{hr} 52 \mathrm{~min}$ & $8 \mathrm{hr} 8 \mathrm{~min}$ \\
\hline
\end{tabular}

The Fitbit monitoring of sleep patterns revealed sleep pattern and time improved with fewer restless periods and reduced awake periods noted. WT reports feeling refreshed on rising daily. While reduced caloric intake was utilized, he met partial success in losing weight with a targeted reduction of 22 pounds. His self-monitoring with the aid of the Fitbit allowed him to control calorie intake along with measuring the benefits of exercise which included walking for designated time periods each day.

(C) The Internet Journal of Allied Health Sciences and Practice, 2016 
While he did not meet expectations and goals in all categories, the subject reported that seeing actual data on his self-monitoring device served to motivate him to continue and meet or exceed the goals that had been set. There were periods where he said he felt somewhat addicted to meeting the measured goals set. This is seen as an important ingredient in using a wearable Smart technology device. The feedback can serve to benefit compliance and continuation in the study activities and continue to guide the subject with healthcare goals agreed upon with allied health care providers.

The subject appears to have benefited from a healthy diet and exercise program wherein the use of the technology may well have played a role. Most people are surprised to find that they have vastly underestimated their caloric intake and overestimated their physical activity. Also, many people overestimate or underestimate their weight problem. Often, seriously overweight people spend much of their lives struggling to lose weight, jumping from one fad diet or new remedy to another without ever achieving lasting success. Utilization of smart technology, along with an effective diet and exercise program, requires professional guidance. Allied health professionals can make all the difference for these patients in achieving improved and recommended health goals including weight, diet, sleep, exercise, self-esteem and an improved quality of life.

\section{DISCUSSION}

The purpose of this study was to determine the effectiveness of a wearable Smart technology device in self-monitoring behavior change with a subject motivated to utilize Smart technology in complying with prescribed outpatient treatment. It was hypothesized that using a wearable self-monitoring device while involved in the treatment of multiple medical conditions may be beneficial to behavioral compliance and behavioral change with a subject being treated by a multidisciplinary integrated treatment team including allied health professionals.

Wearable technology devices, such as the popular Fitbit, have become an enormously popular health and fitness tool since their inception in 2007. Patient and health and wellness enthusiasts benefit from self-tracking devices as they provide a variety of selfmonitoring information and biometrics. When one begins to catch on to self-monitoring various health and wellness factors, one begins to compete with oneself. It can drive many persons to check weight on a daily basis, exercise more, monitor sleep cycles and patterns, and help detect mood shifts over time. When it comes to weight management and losing weight, the main idea behind Fitbit, Nike Fuelband, or the Jawbone is that knowing how many calories one consumes and burns per day makes losing weight easier.

Smart wearable technology devices have alarms that serve as a reminder and aid in monitoring when one forgets to meet goals, needs reminders on certain activities, and exceeds caloric intake against the amount of calories burned through various forms of exercise during the day. For example, one can make sure that he/she hits a daily minimum calories burned that has been set for the patient. Knowing ones overall daily caloric intake and expenditure and how many calories one burns per day via exercise seems to be very useful information to have in meeting goals and understanding one's health profile. Receiving virtual rewards, such as ribbons and badges, was not as motivating as seeing actual numbers. Encouraging people to maintain healthy habits is a challenge that pre-dates technology.

\section{LIMITATIONS}

This study has several limitations. A single case study design is often viewed as lacking size and suffers from the desire for group randomization. With these limitations, the strength of this study is it can serve as a pilot for multiple subject designs with various diagnostic and treatment groups using wearable technology beyond FitBit such as the Smartwatch, Jawbone, Quantified Self, Smart Keychain, and Microsoft Band. Each brings different technology specificity to the allied health clinician researcher.

\section{FUTURE RESEARCH}

As new technology advances for improving healthcare delivery, wearable appliances that form an integral part of our personal life have emerged. Wearable devices can be beneficial in assisting allied health professionals in monitoring effective behavioral changes over time in their patients. It is possible that group interventions will provide similar effects as individual interventions, and should become the focus of further research studies.

\section{CONCLUSION}

Wearable Smart technology may be beneficial in serving as an adjunct to treatment by allied health professionals in self-monitoring behavior change with a subject complying with prescribed outpatient treatment. An exploratory case study research design is employed. It was hypothesized that using a wearable self-monitoring device while involved in the treatment of multiple medical conditions would be beneficial for behavioral compliance and behavioral change in a subject being treated by a multidisciplinary, integrated treatment team. After the 36-week intervention program, results revealed significant qualitative improvements in the active minutes, steps taken, and miles walked by the subject. In addition, the technology reported on calories burned, sleep hours

(C) The Internet Journal of Allied Health Sciences and Practice, 2016 
and minutes logged, and water consumed during each 24-hour period. The integrated allied health team was able to monitor changes made over time and noted improved multiple outcomes.

\section{References}

1. Aarts $\mathrm{H}$, Paulussen $\mathrm{T}$, Schaalma $\mathrm{H}$. Physical exercise habit: on the conceptualization and formation of habitual health behaviours. Health Educ Res. 1997 Sep; 12(3):363-74. [PMID: 10174219]

2. Gusmer RJ, Bosch TA, Watkins AN, Ostrem JD, Dengel DR. Comparison of FitBit ${ }^{\circ}$ Ultra to ActiGraph ${ }^{T M}$ GT1M for assessment of physical activity in young adults during treadmill walking. Open Sports Medicine Journal. 2014;8:11-15.

3. Lee JM, Kim Y,Welk GJ. Validity of consumer-based physical activity monitors. Med Sci Sports Exerc. 2014 Sep; 46(9):18408. [PMID: 24777201]

4. Harrison D, Marshall P, Bianchi-Berthouze N, Bird J. Tracking physical activity: problems related to running longitudinal studies with commercial devices. In: Brush AJ, Friday A, Kientz J, Scott J, Song J. UbiComp '14 Adjunct: Proceedings of the 2014 ACM International Joint Conference on Pervasive and Ubiquitous Computing: Adjunct Publication. 2014, p 699-702.

5. Mammen G, Gardiner S, Senthinathan A, McClemont L, Stone M, Faulkner G. Is this Bit Fit? Measuring the quality of the Fitbit step-counter. The Health \& Fitness Journal of Canada. 2012; 5(4):30-9.

6. Munson SA, Consolvo S. Exploring goal-setting, rewards, self-monitoring, and sharing to motivate physical activity. In Pervasive Computing Technologies for Healthcare (PervasiveHealth), 2012 6th International Conference on (pp. 25-32). IEEE. 2012, May.

7. United States, Office of the Surgeon General 2012 Shape Up America. Washington DC: The U.S. Surgeon General's Guidelines on Promoting Physical Activity among All Americans. Retrieved at: http://www.cdc.gov/nccdphp/sgr/pdf/sgrfull.pdf. Accessed November 21, 2016.

8. Sasaki JE, Hickey A, Mavilia M, Tedesco J, John D, Kozey KS, Freedson PS. Validation of the Fitbit wireless activity tracker® for prediction of energy expenditure. J Phys Act Health. 2015 Feb; 12(2):149-54. [PMID: 24770438]

9. Takacs J, Pollock CL, Guenther JR, Bahar M, Napier C, Hunt MA. Validation of the Fitbit One activity monitor device during treadmill walking. J Sci Med Sport. 2014 Sep; 17(5):496-500. [PMID: 24268570]

10. Wang JB, Cadmus-Bertram LA, Natarajan L, White MM, Madanat H, Nichols JF, Ayala GX, Pierce JP. Wearable sensor/device (Fitbit One) and SMS text-messaging prompts to increase physical activity in overweight and obese adults: a randomized controlled trial. Telemed J E Health. 2015; 21(10):782-92. [PMID: 26431257] 